\title{
EFFECTS OF LINEAR TRANSFORMATIONS ON THE DIVERGENCE OF BOUNDED SEQUENCES AND FUNCTIONS*
}

\author{
BY \\ JOSEPH LEV
}

1. Introduction. The transformation

$$
y_{n}=\sum_{i=1}^{\infty} K_{n, i} x_{i},
$$

where $\left\{x_{i}\right\}$ is a sequence of complex elements and the $K_{n, i}$ are complex numbers, has been widely studied, and the conditions which must be fulfilled by the $K_{n, i}$ in order that the property of convergence of the sequence may remain invariant were given by Schur [1]. $\dagger$ In recent studies by Hurwitz [2,3] and Knopp [4] modes of measuring the divergence of bounded sequences were given, and the conditions on the $K_{n, i}$ were found under which the divergence of the sequence $\left\{y_{n}\right\}$ is no greater than that of $\left\{x_{n}\right\}$.

In this paper the effects of the transformations will be investigated with fewer restrictions on the $K_{n, i}$ than those imposed by earlier writers. The problem will be approached by means of the new concept of the limit circle defined as follows:

The limit circle of a bounded sequence of complex elements is the (unique) circle of least radius which contains within or on its boundary the limit points of the sequence.

The limit circle of a bounded function $F(y)$ of the complex variable $y$ as $y \rightarrow \xi$ (finite or infinite) is analogously defined in terms of the limit points of $F(y)$ as $y \rightarrow \xi$; this concept will be used in the study of transformations of sequences and functions into functions.

2. Sequence to function transformations. Instead of the transformation mentioned in the introduction we shall study the following more general transformation $S$. Let $T$ be a set of points in the complex plane having a limit point $t_{0}$ (finite or infinite) not belonging to $T$. We shall speak of a point $t$ in $T$ as being sufficiently advanced if for some $\delta>0,\left|t-t_{0}\right|<\delta$ when $t_{0}$ is finite, or $|1 / t|<\delta$ when $t_{0}$ is infinite. Then let $K_{i}(t)$ be a set of complex numbers defined for $i=1,2, \cdots$, and each $t$ in $T$, and such that

- Presented to the Society, December 27, 1932; received by the editors November 15, 1932, and after revision, May 17, 1933.

$\dagger$ Here and below numbers in square brackets refer to the bibliography at the end of the paper. 
$S:$

$$
g(t)=\sum_{i=1}^{\infty} K_{i}(t) x_{i}
$$

is defined for each $t$ in $T$. We shall refer to the limit points of $g(t)$ as $t \rightarrow t_{0}$ simply as the limit points of $g(t)$.

We shall now prove

THEOREM 2.1. Let $\left\{x_{n}\right\}$ be a bounded sequence of complex elements. If the $K_{i}(t)$ satisfy the conditions

$$
\begin{gathered}
\lim _{t \rightarrow i_{0}} K_{i}(t)=k_{i}, \text { for each } i, \\
\sum_{i=1}^{\infty}\left|K_{i}(t)\right|<M
\end{gathered}
$$

for all sufficiently advanced $t, M$ a constant, then the quantities $\alpha$ the center and $D$ the radius of the limit circle of the function $\sum_{i=1}^{\infty} K_{i}(t)$,

$$
A=\alpha-\sum_{i=1}^{\infty} k_{i}, B=\sum_{i=1}^{\infty} k_{i} x_{i}, C=\limsup _{i \rightarrow i_{0}} \sum_{i=1}^{\infty}\left|K_{i}(t)-k_{i}\right|
$$

exist, and the limit points of $g(t)$ lie in the circle of center $B=A h+B$, and radius $R=C r+D|h|$, where $h$ is the center and $r$ the radius of the limit circle of $\left\{x_{n}\right\}$.

The existence of $\alpha, A, B, C, D$ is easy to establish and the details will not be given here. For the remainder of the proof write the inequality

$$
\begin{gathered}
\left|\sum_{i=1}^{\infty} K_{i}(t) x_{i}-\sum_{i=1}^{\infty} k_{i} x_{i}-h\left(\alpha-\sum_{i=1}^{\infty} k_{i}\right)\right| \leqq \sum_{i=1}^{\infty}\left|K_{i}(t)-k_{i}\right| \cdot\left|x_{i}-h\right| \\
\quad+\sum_{i=p+1}^{\infty}\left|K_{i}(t)-K_{i}\right| \cdot\left|x_{i}-h\right|+|h| \cdot\left|\sum_{i=1}^{\infty} K_{i}(t)-\alpha\right| .
\end{gathered}
$$

Choose $\epsilon>0$, and $p$ so great that for all $i>p\left|x_{i}-h\right|<r+\epsilon$. Then

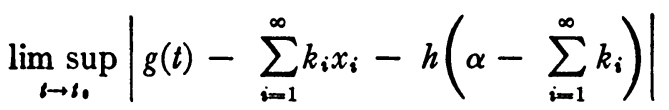

$$
\begin{aligned}
& \leqq o(1)+(r+\epsilon) \cdot \lim \sup _{t \rightarrow i_{0}} \sum_{i=1}^{\infty}\left|K_{i}(t)-k_{i}\right|+|h| \cdot \lim \sup _{t \rightarrow t_{0}}\left|\sum_{i=1}^{\infty} K_{i}(t)-\alpha\right|,
\end{aligned}
$$

and since the inequality holds for all $\epsilon>0$, the theorem follows.

The remaining theorems of this section will be seen to be in part consequences of Theorem 2.1. Notations already introduced will be freely used, 
and $\left\{x_{n}\right\}$ will be taken bounded throughout the discussion. In particular $h$ and $r$ will in each case by taken to depend on $\left\{x_{n}\right\}$.

Theorem 2.1 easily yields the sufficiency of the following theorem of Schur [1].

THEOREM 2.2. In order that $S$ may be such that $\lim _{t \rightarrow t_{0}} g(t)$ exists whenever $h=r=0$, it is necessary and sufficient that the $K_{i}(t)$ satisfy (2.11), and (2.12).

The theorem just stated can be generalized to

THEOREM 2.3. Let $N$ be a real non-negative constant. In order that the limit points of $g(t)$ shall lie in a circle of radius $N|h|$, whenever $r=0$, it is necessary and sufficient that the $K_{i}(t)$ satisfy the conditions (2.11), (2.12), and $D \leqq N$.

The sufficiency follows from Theorem 2.1, and the necessity of the first two conditions from Theorem 2.2. For the necessity of the condition $D \leqq N$ we need only consider the special case $x_{n}=1(n=1,2, \cdots)$.

To supplement Theorem 2.3 we can give the following theorem which takes into account the position of the limit points of $g(t)$.

THEOREM 2.4. Let $N$ be a real non-negative constant. In order that $S$ may be such that the limit points of $g(t)$ shall lie in a circle of center $h$ and radius $N|h|$, whenever $r=0$, it is necessary and sufficient that the $K_{i}(t)$ satisfy, the conditions (2.11), (2.12), $D \leqq N, k_{i}=0$, for all $i$, and $\alpha=1$.

The proof readily follows from a consideration of Theorems 2.1 and 2.3, and, for the necessity of the two last conditions, the sequences $x_{i}=0, i \neq j$, $x_{j}=1$, and the sequence $x_{i}=1(i, j=1,2, \cdots)$.

Obviously the special case $N=0$ yields the well known conditions for regularity, namely the conditions under which $\lim _{t \rightarrow t_{0}} g(t)=\lim _{n \rightarrow \infty} x_{n}$.

We shall now give two theorems which are concerned with divergent sequences.

THEOREM 2.5. Let $Q$ be a real non-negative constant. In order that $S$ may be such that the limit points of $g(t)$ shall lie in a circle of radius $Q r$ whenever $h=0$, it is necessary and sufficient that the $K_{i}(t)$ satisfy the conditions (2.11), (2.12), and $C \leqq Q$.

In the proof we encounter difficulty only in connection with establishing necessity of the condition $C \leqq Q$. We shall assume that (2.11) and (2.12) hold and show that the remaining condition also holds.

Suppose on the contrary $C>Q$. Then for some $\lambda>0$ we have

$$
\sum_{i=1}^{\infty}\left|K_{i}(t)-k_{i}\right|>Q+5 \lambda
$$


repeatedly as $t$ approaches $t_{0}$. There exists, therefore, a sequence $\left\{t_{p}\right\}$ lying entirely in the range for which (2.12) is satisfied, and such that $\lim _{p \rightarrow \infty} t_{p}=t_{0}$; and an increasing sequence of integers $\left\{n_{p}\right\}$ for which the following inequalities hold:

$$
\begin{aligned}
& \sum_{i=1}^{n_{p-1}+1}\left|K_{i}\left(t_{p}\right)-k_{i}\right|<\lambda, \quad \sum_{i=1}^{\infty}\left|K_{i}\left(t_{p}\right)-k_{i}\right|>Q+5 \lambda, \\
& \sum_{i=n_{p}+1}^{\infty}\left|K_{i}\left(t_{p}\right)-k_{i}\right|<\lambda, \quad \sum_{i=n_{p-1}+2}^{n_{p}}\left|K_{i}\left(t_{p}\right)-k_{i}\right|>Q+3 \lambda .
\end{aligned}
$$

In the set $K_{i}\left(t_{p}\right)-k_{i}, n_{p-1}+2 \leqq i \leqq n_{p}$, there is surely one value $K_{q}\left(t_{p}\right)-k_{q}$ which is not zero.

We now define a sequence having a limit circle of center zero and radius one*

$$
\begin{aligned}
x_{i} & =(-1)^{p-1} \operatorname{sgn}\left[K_{i}\left(t_{p}\right)-k_{i}\right], n_{p-1}+2 \leqq i \leqq n_{p}, \\
x_{n_{p}+1} & =(-1)^{p} \operatorname{sgn}\left[K_{\imath}\left(t_{p}\right)-k_{s}\right] .
\end{aligned}
$$

We shall establish the desired contradiction if we show that the limit circle of

$$
g(t)=\sum_{i=1}^{\infty} K_{i}(t) x_{i}
$$

has a radius greater than $Q$. Write

$$
g\left(t_{p}\right)-\sum_{i=1}^{\infty} k_{i} x_{i}=\left(\sum_{i=1}^{n_{p-1}+1}+\sum_{i=n_{p-1}+2}^{n_{p}}+\sum_{i=n_{p}+1}^{\infty}\right)\left[K_{i}\left(t_{p}\right)-k_{i}\right] x_{i} .
$$

The first and third terms on the right are each less than $\lambda$ in absolute value, and the real middle term is greater than $Q+3 \lambda$ for $p$ odd and less than $-(Q+3 \lambda)$ for $p$ even. Hence, writing $R(z)=$ real part of $z$,

$$
\begin{aligned}
R\left[g\left(t_{p}\right)-\sum_{i=1}^{\infty} k_{i} x_{i}\right] & >Q+\lambda, p \text { odd, } \\
& <-(Q+\lambda), p \text { even, }
\end{aligned}
$$

and $g(t)$ has a limit circle of radius greater than $Q$, which completes the proof.

The conditions (2.11), (2.12), and $C \leqq Q$, remain necessary but not sufficient when Theorem 2.5 is written without the hypothesis $h=0$. We can, however, state necessary and sufficient conditions if we restrict ourselves to a consideration of conservative transformations, that is, those transformations

* We use the definition $\operatorname{sgn}(z)=|z| / z, z \neq 0$, and $\operatorname{sgn}(z)=0, z=0$. 
for which $\lim _{t \rightarrow t_{6}} g(t)$ exists whenever $\lim _{n \rightarrow \infty} x_{n}$ exists. The conditions for conservatism are (2.11), (2.12), and $D=0$. Clearly in the conservative case, the condition $C \leqq Q$ is necessary and sufficient for the limit points of $g(t)$ to lie in a circle of radius $Q r$. We can also take into account the position of the limit points and state

THEOREM 2.6. Let $Q$ be a constant, $Q \geqq 1$. In order that the conservative transformation $S$ may be such that the limit points of $g(t)$ shall lie in a circle of radius $Q r$ and center $h$, whenever $\left\{x_{n}\right\}$ is bounded, it is necessary and sufficient that $S$ be regular, and that $C \leqq Q$.

An example due to W. A. Hurwitz yields an interesting comparison between the work of this paper and that of Hurwitz and Knopp. Apply to the sequence $x_{n}=\omega^{2 n}, \omega^{3}=1$, the transformation defined by $K_{n, i}=(-1)^{n} \omega^{i} / 3$ $(i=n, n+1, n+2)$, and $K_{n, i}=0$, otherwise. The resulting sequence, $g_{n}=(-1)^{n}$, has its limit points within the limit circle of the original sequence as is to be expected from our theory but the oscillation of $\left\{g_{n}\right\}$ is greater than that of $\left\{x_{n}\right\}$ and one of its limit points lies outside the limit core of $\left\{x_{n}\right\}$.

3. Function to function transformations. I. In the following let $f(x)$ be a complex function of the real variable $x$ defined and integrable Lebesgue in each interval $a \leqq x \leqq x_{1}<\xi$, where $x_{1}$ is arbitrary and $\xi$ is finite or infinite.

We shall call the following the transformation $S_{1}$. Choose a point set $T$ as in the definition of $S$, and a function $K_{1}(t, x)$ defined for each $t$ in $T$, and each $x, a \leqq x<\xi$, integrable Lebesgue in each interval $a \leqq x \leqq x_{1}<\xi$, for each $t$, such that

$S_{1}$ :

$$
g_{1}(t)=\int_{a}^{\xi} K_{1}(t, s) f(s) d s
$$

exists for each $t$ in $T$.

We shall now give without proof a theorem analogous to Theorem 2.1.

THEOREM 3.1. Let $f(x)$ be bounded $a \leqq x<\xi$. If $S_{1}$ is such that $K_{1}(t, x)$ satisfies the conditions

$$
\begin{gathered}
\lim _{u, t \rightarrow i_{0}} \int_{a}^{x_{1}}\left|K_{1}(t, s)-K_{1}(u, s)\right| d s=0, a \leqq x_{1}<\xi, \\
\int_{a}^{\xi}\left|K_{1}(t, s)\right| d s<M,
\end{gathered}
$$

for all sufficiently advanced $t, M$ a constant, then the quantities $\alpha_{1}$ the center and $D_{1}$ the radius of the limit circle of the function $\int_{a}^{\xi} K_{1}(t, s) d s$, 


$$
\begin{aligned}
& A_{1}=\alpha_{1}-\lim _{x \rightarrow \xi} \lim _{t \rightarrow t_{0}} \int_{a}^{x} K_{1}(t, s) d s, \\
& B_{1}=\lim _{x \rightarrow \xi} \lim _{t \rightarrow t_{0}} \int_{a}^{x} K_{1}(t, s) f(s) d s, \\
& C_{1}=\limsup _{t \rightarrow t_{0}} \lim _{x \rightarrow \xi} \lim _{u \rightarrow t_{0}} \int_{a}^{x}\left|K_{1}(t, s)-K_{1}(u, s)\right| d s
\end{aligned}
$$

exist, and the limit points of $g_{1}(t)$ lie in a circle of center $H_{1}=A_{1} h+B_{1}$, and radius $R_{1}=C_{1} r+D_{1}|h|$, where $h$ is the center and $r$ the radius of the limit circle of $f(x)$.

The sufficiency of theorems analogous to those in $\$ 2$ can easily be established, but for a complete theory analogous to that in $\$ 2$ we need the transformations in the next section.

4. Function to function transformations. II. We shall call the following transformation $S_{2}$. Choose a function $K_{2}(t, x)$ which has all the properties of $K_{1}(t, x)$ and the additional property that $K_{2}(t, x)$ is continuous in $x$, uniformly for all sufficiently advanced $t$, and all $x, a \leqq x \leqq q$, where $q$ is an arbitrary constant less than $\xi$. The transformation is then given by

$S_{2}:$

$$
g_{2}(t)=\int_{a}^{\xi} K_{2}(t, s) f(s) d s .
$$

We can establish

Theorem 4.1. Let $f(x)$ be bounded, $a \leqq x<\xi$. If ' $S_{2}$ is such that $K_{2}(t, x)$ satisfies the conditions

$$
\begin{gathered}
\lim _{t \rightarrow b_{0}} K_{2}(t, x)=k(x), a \leqq x<\xi, \\
\int_{a}^{\xi}\left|K_{2}(t, s)\right| d s<M,
\end{gathered}
$$

for all sufficiently advanced $t, M$ a constant, then the quantities $\alpha_{2}$ the center and $D_{2}$ the radius of the limit circle of the function $\int_{a}^{\xi} K_{2}(t, s) d s$,

$$
\begin{aligned}
& A_{2}=\alpha_{2}-\int_{a}^{\xi} k(s) d s, \quad B_{2}=\int_{a}^{\xi} k(s) f(s) d s, \\
& C_{2}=\limsup _{t \rightarrow i_{0}} \int_{a}^{\xi}\left|K_{2}(t, s)-k(s)\right| d s
\end{aligned}
$$

exist, and the limit points of $g_{2}(t)$ lie in a circle of center $H_{2}=A_{2} h+B_{2}$, and radius $R_{2}=C_{2} r+D_{2}|h|$, where $h$ is the center and $r$ the radius of the limit circle of $f(x)$. 
The proof of this theorem may be made to depend upon that of Theorem 3.1 by showing that $k(x)$ is continuous $a \leqq x<\xi$, that $K_{2}(t, x)$ approaches $k(x)$ uniformly over $a \leqq x \leqq q$, for arbitrary $q$ less than $\xi$, and that $k(x)$ is integrable over $a \leqq x \leqq \xi$. The details will not be given here.

We can now state

THEOREM 4.2. In order that $S_{2}$ may be such that $\lim _{t \rightarrow t_{0}} g_{2}(t)$ exists whenever $h=r=0$, it is necessary and sufficient that $K_{2}(t, x)$ satisfy the conditions (4.11) and (4.12).

The sufficiency follows from Theorem 4.1. The necessity can be established by using the methods of Silverman [5], and Schur [1]; and a consideration of the fact that if $f(x)$ is measurable $a \leqq x \leqq b$, then sgn $f(x)$ is also measurable in this interval.

The remaining analogues of the theorems in $\$ 2$ can easily be stated and proved by methods suggested in that section, and will not be given here.

5. Bounds of the sets of limit points. It is easy to see that in parts of our discussion we can replace the limit circle by some other circle which contains the limit points of the sequence or function. In particular we can replace it by a circle with center at the origin and radius equal to the maximum of the distances from the origin to the limit points. This radius which is a bound for the set of limit points may be written in the case of sequences as lim $\sup _{n \rightarrow \infty}$ $\left|x_{n}\right|$. We can state

THEOREM 5.1. Let $Q$ be a real non-negative constant. In order that

$$
\limsup _{t \rightarrow t_{0}}|g(t)| \leqq Q \limsup _{n \rightarrow \infty}\left|x_{n}\right|
$$

whenever $\left\{x_{n}\right\}$ is bounded, it is necessary and sufficient that the $K_{i}(t)$ satisfy the conditions (2.11), (2.12), $C \leqq Q$, and $k_{i}=0$, for all $i$.

For the proof of necessity consider Theorem 2.5 and, for the last condition, the sequence $x_{i}=0(i=1,2, \cdots)$; and the sequences $x_{j}=1, x_{i}=0$, $i \neq j(i, j=1,2, \cdots)$.

6. Application to series. We shall generalize some results due to Schur [1] and Kojima [6].

Let the series $w_{0}+w_{1}+w_{2}+\cdots$, with partial sums $W_{n}$, be the Cauchy product of the two series $u_{0}+u_{1}+u_{2}+\cdots$, and $v_{0}+v_{1}+v_{2}+\cdots$, with partial sums $U_{n}$ and $V_{n}$ respectively. We can write

$$
W_{n}=u_{n} V_{0}+\cdots+u_{0} V_{n},
$$

which is a linear transformation on the sequence $\left\{V_{n}\right\}$. If we suppose that $\sum\left|u_{n}\right|$ converges and that $\left\{V_{n}\right\}$ is bounded with limit circle of center $h$ and 
radius $r$ we can apply Theorem 2.1 to show that the limit points of $\left\{W_{n}\right\}$ lie in a circle of center $h \sum u_{n}$ and radius $r \sum\left|u_{n}\right|$.

Now write $U_{n}^{p}=\sum_{i=0}^{n} A_{n-i}^{p} u_{i}$, where $A_{n}^{p}=\Gamma(p+n+1) /[\Gamma(n+1) \Gamma(p+1)]$ and $p \geqq 0$. If the Cesàro transform of order $p, C_{n}^{p}(u)=U_{n}^{p} / A_{n}^{p}$, is bounded, the series $\sum u_{n}$ is said to be bounded $(C, p)$. Writing similar expressions for the series $\sum v_{n}$ and $\sum w_{n}$ we get $W_{n}^{p+q+1}=\sum_{i=0}^{n} V_{n-i}^{q} U_{i}^{p}, q \geqq 0$, which may be written

$$
C_{n}^{p+q+1}(w)=\frac{1}{A_{n}^{p+q+1}} \sum_{i=0}^{n} A_{i}^{p} V_{n-i}^{q} C_{i}^{p}(u) .
$$

If we regard this expression as a linear transformation on the $C_{i}^{p}(u)$ we get $K_{n, i}=A_{i}^{p} V_{n-i}^{q} / A_{n}^{p+q+1}(i=0,1,2, \cdots, n), K_{n, i}=0(i>n)$. Then supposing that $\sum v_{n}$ is bounded $(C, q)$, we have $\left|K_{n, i}\right|=A_{i}^{p} A_{n-i}^{q}\left|C_{n-i}(v)\right|$ $/ A_{u}^{p+q+1}<M / n^{p+1}, M$ a constant for all $n$, so that $\lim _{n \rightarrow \infty} K_{n, i}=0$. Furthermore $\sum_{i=0}^{n}\left|K_{n, i}\right|<N, N$ a constant for all $n$, and $\sum_{i=0}^{n} K_{n, i}=C_{n}^{p+q+1}(v)$. Hence if we call the center and radius of $\left\{C_{n}^{p}(u)\right\}$, and $\left\{C_{n}^{p+q+1}(v)\right\}, h_{u}$, $r_{u}$, and $h_{v}, r_{v}$, respectively, we have

TheOREM 6.1. If $\sum u_{n}$ is bounded $(C, p)$ and $\sum v_{n}$ is bounded $(C, q), p, q \geqq 0$, then the sequence $\left\{C_{n}^{p+q+1}(w)\right\}$ has its limit points in a circle of center $h_{v} h_{u}$, and radius $r_{u} \cdot \lim \sup _{n \rightarrow \infty} \sum_{i=0}^{n} A_{i}^{p}\left|V_{n-i}^{q}\right| / A_{n}^{p+q+1}+r_{v}\left|h_{u}\right|$.

If we consider the two series $\sum u_{n}$ with partial sums $s_{n}$, and $\sum c_{n} u_{n}$ with partial sums $t_{n}$ we get

$$
t_{n}=s_{0}\left(c_{0}-c_{1}\right)+\cdots+s_{n-1}\left(c_{n-1}-c_{n}\right)+s_{n} c_{n} .
$$

On the basis of the assumptions that $\left\{s_{n}\right\}$ is bounded with limit circle of center $h$ and radius $r$, and that $\sum_{n=0}^{\infty}\left|c_{n}-c_{n+1}\right|$ converges, we can show by means of Theorem 2.1 that the limit points of $\left\{t_{n}\right\}$ lie in a circle of center $h \cdot \lim _{n \rightarrow \infty} c_{n}+\sum_{n=0}^{\infty}\left(c_{n}-c_{n+1}\right) s_{n}$, and radius $r \cdot \lim _{n \rightarrow \infty}\left|c_{n}\right|$.

Generalizations of the last result to the case when $\sum u_{n}$ is bounded $(C, p)$ for some $p>0$ can easily be arrived at on the basis of the work of Schur [1] and Kojima [6].

\section{BIBLIOGRAPHY}

1. J. Schur, Über lineare Transformationen in der Theorie der unendlichen Reihen, Journal für die reine und angewandte Mathematik, vol. 151 (1920), pp. 79-111.

2. W. A. Hurwitz, Some properties of methods of evaluation of divergent sequences, Proceedings of the London Mathematical Society, (2), vol. 26 (1927), pp. 231-248. 
3. W. A. Hurwitz, The oscillation of a sequence, American Journal of Mathematics, vol. 52 (1930), pp. 611-616.

4. K. Knopp, Zur Theorie der Limitierungsverfahren, Mathematische Zeitschrift, vol. 31 (1930), pp. 97-127, and 276-305.

5. L. L. Silverman, On the notion of summability for the limit of a function of a real variable, these Transactions, vol. 17 (1916), pp. 284-294.

6. T. Kojima, On generalized Toeplitz's theorem on limits, Tôhoku Mathematical Journal, vol. 12 (1917), pp. 291-326.

CORNEIL UNIVERSTTY,

ITHACA, N. Y. 